\title{
Quantum phase transition in an atomic Bose gas with a Feshbach resonance
}

\author{
M.W.J. Romans, ${ }^{1}$ R.A. Duine, ${ }^{1}$ Subir Sachdev, ${ }^{2}$ and H.T.C. Stoof ${ }^{1}$ \\ ${ }^{1}$ Institute for Theoretical Physics, Utrecht University, \\ Leuvenlaan 4, 3584 CE Utrecht, The Netherlands \\ ${ }^{2}$ Department of Physics, Yale University, P.O. Box 208120, New Haven, Connecticut 06520-8120, USA
}

\begin{abstract}
We show that in an atomic Bose gas near a Feshbach resonance a quantum phase transition occurs between a phase with only a molecular Bose-Einstein condensate and a phase with both an atomic and a molecular Bose-Einstein condensate. We show that the transition is characterized by an Ising order parameter. We also determine the phase diagram of the gas as a function of magnetic field and temperature: the quantum critical point extends into a line of finite temperature Ising transitions.
\end{abstract}

Introduction. - One of the most important recent developments in the field of ultracold atomic gases is the application of Feshbach resonances. A Feshbach resonance in the scattering amplitude of two atoms occurs when the total energy of the atoms is close to the energy of a molecular state that is weakly coupled to the atomic continuum [1, 2]. In the alkali gases of interest, this coupling is provided by the exchange interaction, and consequently the magnetic moments of the two atoms and the molecule differ substantially. As a result the energy difference between the molecule and the threshold of the two-atom continuum, known as the detuning $\delta$, can be experimentally tuned by means of a magnetic field. Moreover, by sweeping the magnetic field from positive to negative detuning through the Feshbach resonance, it is actually possible to form molecules in the atomic gas [3, 4, 5, 6, 7].

Very recently, it has even been possible to create a Bose-Einstein condensate (BEC) of molecules in an atomic Fermi gas with a Feshbach resonance [8, 9, 10]. This achievement is of particular importance because it offers the opportunity for observing a Bardeen-CooperSchrieffer (BCS) transition in a dilute atomic gas 11, 12]. The idea here is to start from a pure molecular condensate and then sweeping the magnetic field back to positive detuning to create a much colder atomic Fermi gas than could have been achieved without the intermediate step of forming a molecular Bose-Einstein condensate. It is important to realize that this idea strongly hinges on the fact that a smooth BEC-BCS crossover exists in this system as one changes $\delta[13,14,15]$. In this Letter we show that analogous experiments varying $\delta$ in an atomic Bose lead to a true phase transition rather than a crossover [16]. The reason for, and the nature of, this phase transition can be understood as follows.

The argument contains two important ingredients. The first ingredient is that the coupling between the atoms and molecules in the gas is provided by an interaction energy that is proportional to $\int d \mathbf{x}\left(\psi_{\mathrm{m}}^{\dagger}(\mathbf{x}) \psi_{\mathrm{a}}(\mathbf{x}) \psi_{\mathbf{a}}(\mathbf{x})+\psi_{\mathrm{a}}^{\dagger}(\mathbf{x}) \psi_{\mathrm{a}}^{\dagger}(\mathbf{x}) \psi_{\mathrm{m}}(\mathbf{x})\right)$, where $\psi_{\mathrm{a}}(\mathbf{x})$ and $\psi_{\mathrm{m}}(\mathbf{x})$ annihilate an atom and a molecule at position $\mathbf{x}$, respectively [17, 18, 19]. Such a coupling implies that if the gas contains an atomic Bose-Einstein condensate, and therefore has a nonzero value of $\left\langle\psi_{\mathrm{a}}(\mathbf{x})\right\rangle$, the gas must necessarily also contain a molecular BoseEinstein condensate and have a nonzero value of $\left\langle\psi_{\mathrm{m}}(\mathbf{x})\right\rangle$. However, the reverse is not true, and it is possible for the gas to contain only a molecular Bose-Einstein condensate.

The second ingredient is that the symmetries of these two different phases of the gas are different. In the normal phase the gas is invariant under the phase transformations $\psi_{\mathrm{a}}(\mathbf{x}) \rightarrow e^{i \theta} \psi_{\mathrm{a}}(\mathbf{x})$ and $\psi_{\mathrm{m}}(\mathbf{x}) \rightarrow e^{2 i \theta} \psi_{\mathrm{m}}(\mathbf{x})$. The additional factor of two in the transformation of the molecular annihilation operator follows also from the above interaction energy and is physically related to the fact that an molecule consist of two atoms. If the gas contains both an atomic and a molecular BoseEinstein condensate $(\mathrm{AC}+\mathrm{MC})$ this $U(1)$ symmetry is completely broken, and no residual symmetry exists. However, if the gas contains only a molecular condensate (MC), a residual discrete symmetry remains because $\left\langle\psi_{\mathrm{m}}(\mathbf{x})\right\rangle \rightarrow\left\langle\psi_{\mathrm{m}}(\mathbf{x})\right\rangle$ for $\theta=\pi$. This phase therefore only breaks the $U(1) / Z_{2}$ symmetry spontaneously. As a result there must exist an Ising-like phase transition between the state with only a $\mathrm{MC}$ and the state with an $\mathrm{AC}+\mathrm{MC}$, in which the residual $Z_{2}$ symmetry is spontaneously broken. Note that in the case of an atomic Fermi gas the BCS phase is characterized by a nonzero value of $\left\langle\psi_{\mathbf{a}}(\mathbf{x}) \psi_{\mathbf{a}}(\mathbf{x})\right\rangle$. The BCS phase has therefore the same symmetry as a molecular Bose-Einstein condensate and only a crossover occurs.

The existence of the Ising transition for the case of the Bose gas also becomes clear from a consideration of the allowed vortices in the two limiting cases. The vortices in the state with only a MC are quantized in integer multiples of an elementary circulation which is exactly one half the quantum of circulation in the state with an $\mathrm{AC}+\mathrm{MC}$. Consequently there is a deconfinement-toconfinement transition for a pair of elementary vortices in the MC with increasing detuning [20]. Experiments in the atomic Bose gases thus have the prospect of observing this phase transition with an essentially topological character, something which has not so far been possible in condensed-matter systems.

The phase diagram that we expect on the basis of the 
above arguments is shown in Fig. 1] For large positive $\delta$ the molecular energy lies far above the threshold of the two-atom continuum and the gas consists essentially completely of atoms, which Bose-Einstein condense approximately at the ideal gas critical temperature $T=T_{0} \equiv\left(2 \pi \hbar^{2} / m k_{\mathrm{B}}\right)[n / \zeta(3 / 2)]^{2 / 3}$, where $n$ is the total atomic density of the gas that is assumed to be a constant throughout the phase diagram. If we lower $\delta$ towards zero, the number of molecules in the gas increases and consequently the critical $T$ decreases monotonously. For large negative $\delta$, the molecular energy lies far below the threshold of the two-atom continuum, and the gas consists solely of molecules that condense at the critical temperature $T_{0} / 2^{5 / 3}$. Upon increasing $\delta$ towards zero, the number of atoms in the gas increases, and the critical $T$ for Bose-Einstein condensation decreases again. Finally, our arguments have shown that by increasing $\delta$ at a fixed $T$ below the critical $T$ for Bose-Einstein condensation of the molecules, a Ising phase transition to a phase with both an atomic and a molecular Bose-Einstein condensate occurs. This occurs always at a negative $\delta$, as we will see next, when we discuss in detail the quantitative determination of the phase diagram.

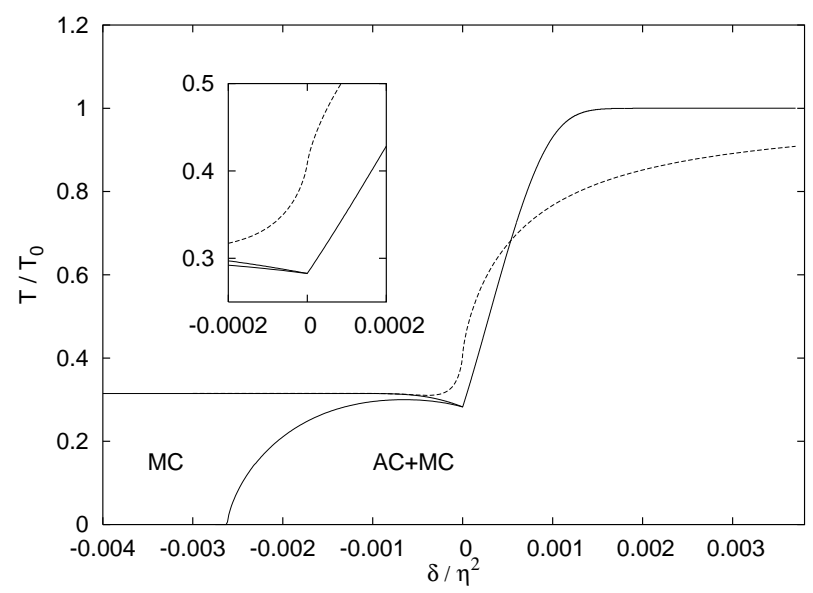

FIG. 1: Phase diagram of a Bose gas with $n=10^{13} \mathrm{~cm}^{-3}$ near a Feshbach resonance as a function of detuning $\delta$ and temperature $T$. The solid lines represents the critical $T$ for the transitions to the normal state, and the location of the Ising transition between the phase with only a molecular Bose-Einstein condensate (MC) and the phase with both an atomic and a molecular Bose-Einstein condensate $(\mathrm{AC}+\mathrm{MC})$. The solid lines do not include the effects of the finite lifetime of the molecules. The dashed line shows a calculation of the critical $T$ for the transitions to the normal state that includes these effects.

Negative detuning. - Up to now we have not made a careful distinction between bare and dressed molecules. However, for a realistic calculation of the phase diagram this distinction is very important. The bare molecule corresponds to the bound state of the Feshbach problem in the absence of a coupling with the two-atom contin- uum, whereas the dressed molecule corresponds to the bound state of the full problem including that coupling. As a result the energy of the dressed molecule is not equal to the detuning, but turns out to be given by $\epsilon_{\mathrm{m}}=\delta+\eta^{2}\left(\sqrt{1-4 \delta / \eta^{2}}-1\right) / 2$, where $\eta^{2}=g^{4} m^{3} / 4 \pi^{2} \hbar^{6}$ and $g=\hbar \sqrt{2 \pi a_{\mathrm{bg}} \Delta B \Delta \mu / m}$ determines the coupling between the atoms and the bare molecules [21]. The latter can be expressed in terms of the background scattering length $a_{\mathrm{bg}}$, the magnetic field width $\Delta B$, and the difference between the magnetic moments of a bare molecule and two atoms $\Delta \mu$, which are the experimentally know parameters of the Feshbach resonance. Furthermore, the wave function of the dressed molecule is not equal to the wave function of the bare molecule, but contains also amplitude to be in the two-atom continuum. In detail we find 22]

$$
\left.\left.\left.\mid \chi_{\mathrm{m}} ; \text { dressed }\right\rangle=\sqrt{Z} \mid \chi_{\mathrm{m}} ; \text { bare }\right\rangle+\sum_{\mathbf{k}} C_{\mathbf{k}} \mid \mathbf{k},-\mathbf{k} ; \text { open }\right\rangle,
$$

where the wave-function renormalization factor $Z$ obeys $1 / Z=1+\eta / 2 \sqrt{\left|\epsilon_{m}\right|}$. Far from resonance we have that $Z \simeq 1$ and there is essentially no distinction between the bare and dressed molecular wave functions. However, close to resonance $Z$ becomes very small and the dressed molecule actually has almost no amplitude to be in the bare molecular state.

To take the above physics most easily into account we introduce creation and annihilation operators for the dressed molecules. The grand-canonical hamiltonian for the gas then becomes

$$
\begin{aligned}
H & =\int d x \psi_{\mathrm{m}}^{\dagger}(x)\left[-\frac{\hbar^{2} \nabla^{2}}{4 m}+\epsilon_{\mathrm{m}}-2 \mu\right] \psi_{\mathrm{m}}(x) \\
& +\int d x \psi_{\mathrm{a}}^{\dagger}(x)\left[-\frac{\hbar^{2} \nabla^{2}}{2 m}-\mu+\frac{T_{\mathrm{bg}}}{2} \psi_{\mathrm{a}}^{\dagger}(x) \psi_{\mathrm{a}}(x)\right] \psi_{\mathrm{a}}(x) \\
& +\int d x \sqrt{Z} g\left[\psi_{\mathrm{m}}^{\dagger}(x) \psi_{\mathrm{a}}(x) \psi_{\mathrm{a}}(x)+\psi_{\mathrm{a}}^{\dagger}(x) \psi_{\mathrm{a}}^{\dagger}(x) \psi_{\mathrm{m}}(x)\right] \\
& +\int d x \frac{T_{\mathrm{mm}}}{2} \psi_{\mathrm{m}}^{\dagger}(x) \psi_{\mathrm{m}}^{\dagger}(x) \psi_{\mathrm{m}}(x) \psi_{\mathrm{m}}(x) \\
& +\int d x T_{\mathrm{am}} \psi_{\mathrm{m}}^{\dagger}(x) \psi_{\mathrm{a}}^{\dagger}(x) \psi_{\mathrm{a}}(x) \psi_{\mathrm{m}}(x),
\end{aligned}
$$

where $T_{\mathrm{bg}}=4 \pi a_{\mathrm{bg}} \hbar^{2} / m$, and $T_{\mathrm{am}}$ and $T_{\mathrm{mm}}$ are the $\mathrm{T}$ matrices for the scattering of an atom with a dressed molecule and for the scattering of two dressed molecules, respectively. Near resonance the latter two T matrices can actually be expressed in terms of the full atomic scattering length $a(B)=a_{\mathrm{bg}}\left[1-\Delta B /\left(B-B_{0}\right)\right]$ of the Feshbach resonance at magnetic field $B_{0}$ as we show at the end of this Letter.

To find the phase diagram for negative $\delta$, we consider the phase with only a Bose-Einstein condensate of dressed molecules, and perform a quadratic expansion of the hamiltonian around the nonzero expectation value 
$\left\langle\psi_{\mathrm{m}}(\mathbf{x})\right\rangle \equiv \sqrt{n_{\mathrm{mc}}}$. For atoms with zero momentum, the resulting hamiltonian leads to a fluctuation matrix given by

$$
\left[\begin{array}{cc}
-\epsilon_{\mathrm{m}}+\left(2 T_{\mathrm{am}}-T_{\mathrm{mm}}\right) n_{\mathrm{mc}} & 4 g \sqrt{Z} \sqrt{n_{\mathrm{mc}}} \\
4 g \sqrt{Z} \sqrt{n_{\mathrm{mc}}} & -\epsilon_{\mathrm{m}}+\left(2 T_{\mathrm{am}}-T_{\mathrm{mm}}\right) n_{\mathrm{mc}}
\end{array}\right] .
$$

This fluctuation matrix has two positive eigenvalues only if $\delta$ is sufficiently negative or, more precisely, if $\epsilon_{\mathrm{m}}<$ $-4 g \sqrt{Z} \sqrt{n_{\mathrm{mc}}}+\left(2 T_{\mathrm{am}}-T_{\mathrm{mm}}\right) n_{\mathrm{mc}}$. For larger $\delta$ an instability arises, which physically leads to the formation of an atomic condensate in addition to the molecular condensate that is already present. This condition therefore determines the position of the quantum (at $T=0$ ) or classical (for $T>0$ ) Ising phase transition. The critical $T$ for the Bose-Einstein condensation of the dressed molecules is of course obtained from the condition $n_{\mathrm{mc}}=0$.

Since both conditions are expressed in terms of the molecular condensate density $n_{m c}$, we now need to find the equation of state of the gas to obtain this condensate density as a function of $\delta$ and $T$. This is complicated by the fact that the total atomic density of the gas is given by the sum of the density of atoms and of twice the density of bare molecules, i.e., not twice the density of the dressed molecules. Using the techniques derived in Ref. 19 the calculation can nevertheless be performed and we find that in a good approximation the total density of bare molecules is

$$
\begin{aligned}
n_{\mathrm{m}} & =Z n_{\mathrm{mc}}+\frac{1}{V} \sum_{\mathbf{k}}\left(\frac{\epsilon_{\mathbf{k}}+2 T_{\mathrm{mm}} n_{\mathrm{mc}}}{2 \hbar \omega_{\mathbf{k}}} \frac{1}{e^{\beta \hbar \omega_{\mathbf{k}}}-1}\right. \\
& \left.+\frac{\epsilon_{\mathbf{k}}+2 T_{\mathrm{mm}} n_{\mathrm{mc}}-2 \hbar \omega_{\mathbf{k}}}{4 \hbar \omega_{\mathbf{k}}}\right),
\end{aligned}
$$

with $\epsilon_{\mathbf{k}}=\hbar^{2} \mathbf{k}^{2} / 2 m$ and $\hbar \omega_{\mathbf{k}}=\sqrt{\epsilon_{\mathbf{k}}^{2} / 4+\epsilon_{\mathbf{k}} T_{\mathrm{mm}} n_{\mathrm{mc}}}$ the molecular Bogoliubov dispersion. For the atomic density we find in a similar manner that

$$
\begin{aligned}
n_{\mathrm{a}} & =\frac{1}{V} \sum_{\mathbf{k}}\left(\frac{2 \epsilon_{\mathbf{k}}-\epsilon_{\mathrm{m}}+\left(2 T_{\mathrm{am}}-T_{\mathrm{mm}}\right) n_{\mathrm{mc}}}{2 \hbar \omega_{\mathbf{k}}} \frac{1}{e^{\beta \hbar \omega_{\mathbf{k}}}-1}\right. \\
& \left.+\frac{2 \epsilon_{\mathbf{k}}-\epsilon_{\mathrm{m}}+\left(2 T_{\mathrm{am}}-T_{\mathrm{mm}}\right) n_{\mathrm{mc}}-\hbar \omega_{\mathbf{k}}}{4 \hbar \omega_{\mathbf{k}}}\right)
\end{aligned}
$$

where the dispersion for the atoms obeys $\hbar \omega_{\mathbf{k}}=$ $\sqrt{\left[\epsilon_{\mathbf{k}}-\epsilon_{\mathrm{m}} / 2+\left(T_{\mathrm{am}}-T_{\mathrm{mm}} / 2\right) n_{\mathrm{mc}}\right]^{2}-4 g^{2} Z n_{\mathrm{mc}}}$. Note that this dispersion in general has a gap, but becomes gapless exactly at the critical condition for the Ising transition, as expected.

Positive detuning. - At positive $\delta$ no truly stable molecular state exists and the previous approach in principle does not apply. That approach was based on the fact that for negative $\delta$, the density of states for the bare molecules contained a delta function at the energy $\epsilon_{\mathrm{m}}$ and with strength $Z$, that corresponded to the dressed molecules. For positive $\delta$ the density of states consists, however, only of a single broad peak 21]. Approximating that broad peak by a delta function at the energy where the density of states has a maximum, we can again use the hamiltonian in Eq. (2), but now with

$$
\epsilon_{\mathrm{m}}=\frac{1}{3}\left(\delta-\frac{\eta^{2}}{2}+\sqrt{\frac{\eta^{4}}{4}-\eta^{2} \delta+4 \delta^{2}}\right)=\frac{\delta^{2}}{\eta^{2}}+O\left(\delta^{3}\right)
$$

and $Z=1$. In this approximation we are neglecting the finite lifetime of the bare molecules, that is due to the fact that these molecules can now decay into the twoatom continuum. At the end of this Letter we, however, also discuss a calculation that includes these finite lifetime effects exactly [19]. The result of that calculation is indicated by the dashed line in Fig. 1, which shows that the above approximation is indeed rather accurate. Since for positive $\delta$ we only need to determine the critical $T$ for Bose-Einstein condensation of the atoms and molecules, we here consider only the normal phase of the gas. At the critical temperature $T_{\mathrm{c}}$ the atomic density is therefore just $n_{\mathrm{a}}=n\left(T_{\mathrm{c}} / T_{0}\right)^{3 / 2}$, whereas the density of molecules equals

$$
n_{\mathrm{m}}=2 \sqrt{2} n\left(\frac{T_{\mathrm{c}}}{T_{0}}\right)^{3 / 2} \frac{g_{3 / 2}\left(e^{-\beta \epsilon_{\mathrm{m}}}\right)}{\zeta(3 / 2)},
$$

where $g_{3 / 2}(z)$ is the usual Bose function. The desired critical $T$ now follows from $n=2 n_{\mathrm{m}}+n_{\mathrm{a}}$.

Discussion and conclusions. - Up to now we have left the molecule-molecule interaction and the atom-molecule interaction unspecified. Near resonance they are however in first approximation determined by the Feynman diagrams given in Fig. 2 Evaluating these diagrams at zero external momenta and frequencies, we find that $T_{\mathrm{am}}=8 g^{2} Z /\left|\epsilon_{\mathrm{m}}\right|$. Close to resonance we have that $Z=$ $4 \pi \hbar^{4} / g^{2} m^{2} a$ and $\left|\epsilon_{m}\right|=\hbar^{2} / m a^{2}$. We thus conclude that the scattering length for this process is proportional to the atom-atom scattering length as $a_{\text {am }}=32 a / 3$. Using a similar procedure, the scattering length for the moleculemolecule interaction can be shown to be $a_{\mathrm{mm}}=4 a$. In the case of an atomic Fermi gas we find in the same manner that $a_{\mathrm{am}}=8 a / 3$ and $a_{\mathrm{mm}}=a$. This may be compared to similar results obtained recently by Petrov et al. for this case [23].

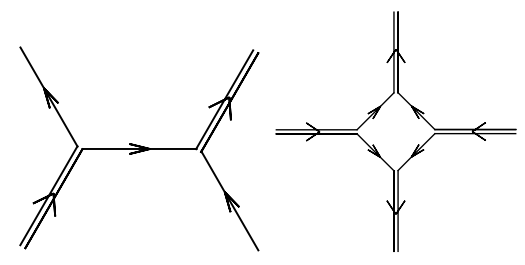

FIG. 2: Feynman diagrams that determine a) the interaction between an atom and a dressed molecule and b) the interaction between two dressed molecules. 
In the calculation of the phase diagram presented in Fig. 11 we have actually not included the mean-field effects due to the atom-molecule and molecule-molecule interactions. The reason is that these mean-field effects, if we also take into account the unitarity limit of the $\mathrm{T}$ matrix, are estimated to lead only to relatively small shifts. Qualitatively, the most important effect is that the Ising transition is, near $T=0$, shifted to somewhat higher values of $\delta$. In particular, the transition temperatures for Bose-Einstein condensation are hardly affected. As mentioned previously, we have performed a calculation of the critical $T$ for Bose-Einstein condensation as a function of $\delta$, that includes both the effects of the finite lifetime of the molecule at positive $\delta$, as well as the rogue-dissociation process 24 for negative $\delta$. The results are presented by the dashed line in Fig. 11 Note that for negative $\delta$ these effects are nonnegligible only in a small region close to resonance, due to the fact that $Z$ becomes very small, which implies that there is a rather large spectral weight in the density of states at positive energies. In addition, $\epsilon_{\mathrm{m}}$ becomes very small, which implies that the positive energy states also get thermally populated. For positive $\delta$ the results are affected more, because of the fact that the finite lifetime of the molecules leads to a substantial broadening of the density of states, which considerably affects the total number of bare molecules in the gas. In future work we intend to determine also the effects of rogue dissociation on the critical line of the Ising transition. We also want to explore the possibility that sufficiently close to resonance, the continuous Ising transition is actually preempted by a first-order phase transition by such effects.

Field theories for critical fluctuations near the Ising transitions at $T=0$ and $T>0$ can be developed by standard methods, using that in the states with $\left\langle\psi_{\mathrm{m}}\right\rangle \neq 0$ the Ising order parameter $\phi \propto i\left(\psi_{\mathrm{a}}-\psi_{\mathrm{a}}^{\dagger}\right)$. These field theories have the familiar $\phi^{4}$ form, except that at $T=0$ there is also a marginal coupling to dynamic density fluctuations of the superfluid which apparently drives the transition weakly first-order [25] (the $T>0$ transition can remain second order). The critical precursors of the transition lead to a large density of states for collective low energy excitations, which will strongly damp single-particle excitations. We speculate that observation of such enhanced sources of dissipation may serve, in addition to direct observation of the $\mathrm{MC}$ and $\mathrm{AC}+\mathrm{MC}$, as an experimental signature of the Ising transition.

While the writing of this paper was being completed, we became aware of the work of Radzihovsky et al. [26], who also point out the existence of the Ising phase transition, but use the Timmermans model [18] to determine the phase diagram. This work is supported by the Stichting voor Fundamenteel Onderzoek der Materie (FOM), the Nederlandse Organisatie voor Wetenschaplijk Onder- zoek (NWO), and the US National Science Foundation grant DMR-0098226.

[1] W. C. Stwalley, Phys. Rev. Lett. 37, 1628 (1976).

[2] E. Tiesinga, B. J. Verhaar, and H. T. C. Stoof, Phys. Rev. A 47, 4114 (1993).

[3] C. A. Regal, C. Ticknor, J. L. Bohn, and D. S. Jin, Nature 424, 47 (2003).

[4] K. E. Strecker, G. B. Partridge, and R. G. Hulet, Phys. Rev. Lett. 91, 080406 (2003).

[5] J. Cubizolles, T. Bourdel, S. J. J. M. F. Kokkelmans, G. V. Shlyapnikov, C. Salomon (cond-mat/0308018).

[6] S. Jochim, M. Bartenstein, A. Altmeyer, G. Hendl, C. Chin, J. Hecker Denschlag, R. Grimm, (cond-mat/0308095).

[7] K. Xu, T. Mukaiyama, J. R. Abo-Shaeer, J. K. Chin, D. Miller, W. Ketterle, Phys. Rev. Lett. 91, 210402 (2003).

[8] S. Jochim, M. Bartenstein, A. Altmeyer, G. Hendl, S. Riedl, C. Chin, J. Hecker Denschlag, and R. Grimm, Science 1093280 (2003).

[9] M. Greiner, C. A. Regal, and D. S. Jin (cond-mat/0311172).

[10] M. W. Zwierlein, C. A. Stan, C. H. Schunck, S. M. F. Raupach, S. Gupta, Z. Hadzibabic, W. Ketterle (cond-mat/0311617).

[11] H. T. C. Stoof, M. Houbiers, C. A. Sackett, and R. G. Hulet, Phys. Rev. Lett. 76, 10 (1996)

[12] M. Houbiers, R. Ferwerda, H. T. C. Stoof, W. I. McAlexander, C. A. Sackett, and R. G. Hulet, Phys. Rev. A 56, 4864 (1997).

[13] Y. Ohashi and A. Griffin, Phys. Rev. Lett. 89, 130402 (2002).

[14] Y. Ohashi and A. Griffin, Phys. Rev. A 67, 033603 (2003); Phys. Rev. A 67, 063612 (2003).

[15] J. N. Milstein, S. J. J. M. F. Kokkelmans and M. J. Holland, Phys. Rev. A 66, 043604 (2002).

[16] A transition appears in the variational computation of P. Nozieres and D. Saint James, J. Physique 43, 1133 (1982), but no statements were made on the generality and nature of this transition.

[17] P. D. Drummond, K. V. Kheruntsyan, and H. He, Phys. Rev. Lett. 81, 3055 (1998).

[18] E. Timmermans, P. Tommasini, H. Hussein, and A. Kerman, Phys. Rep. 315, 199 (1999).

[19] R. A. Duine and H. T. C. Stoof (cond-mat/0308384).

[20] In three dimensions at nonzero temperatures, a duality mapping shows that this is the confinement transition in a $Z_{2}$ gauge theory that is dual to the Ising model.

[21] R. A. Duine and H. T. C. Stoof, J. Opt. B: Quantum Semiclass. Opt. 5, S212 (2003).

[22] R. A. Duine and H. T. C. Stoof, Phys. Rev. A 68, 013602 (2003); Phys. Rev. Lett. 91, 150405 (2003).

[23] D. S. Petrov, C. Salomon and G. V. Shlyapnikov (cond-mat/0309010).

[24] M. Mackie, K.-A. Suominen, and J. Javanainen, Phys. Rev. Lett. 89, 180403 (2002).

[25] E. Frey and L. Balents, Phys. Rev. B 55, 1050 (1997).

[26] L. Radzihovsky, J. Park, P. Weichman (cond-mat/0312237). 